PROCEEDINGS OF THE

AMERICAN MATHEMATICAL SOCIETY

Volume 129, Number 10, Pages 2907-2911

S 0002-9939(01)05889-0

Article electronically published on February 15, 2001

\title{
ON THE DEFINITION OF VISCOSITY SOLUTIONS FOR PARABOLIC EQUATIONS
}

\author{
PETRI JUUTINEN
}

(Communicated by Albert Baernstein II)

\begin{abstract}
In this short note we suggest a refinement for the definition of viscosity solutions for parabolic equations. The new version of the definition is equivalent to the usual one and it better adapts to the properties of parabolic equations. The basic idea is to determine the admissibility of a test function based on its behavior prior to the given moment of time and ignore what happens at times after that.
\end{abstract}

\section{INTRODUCTION}

Consider the second order parabolic equation

$$
u_{t}+F\left(x, t, u, D u, D^{2} u\right)=0 .
$$

Here, as usual, $D u$ denotes the spatial gradient and $D^{2} u$ is used for the corresponding $n \times n$ matrix of the second derivatives. Assuming only that $F$ is continuous and proper, equations of this type can be treated using the technology of the viscosity solutions developed by Crandall, Lions, Ishii, Evans, Jensen and many others (see [CIL]). In the standard viscosity theory, parabolic equations are basically seen as if they were elliptic equations of a specific form and the definition of a solution follows this logic. In this note we propose a different approach. Rather than just thinking of $(\mathrm{P})$ as an equation of $n+1$ independent variables we wish to emphasize the special role of the $t$ variable: at least heuristically, what happens at $\left(x_{0}, t_{0}\right)$ is completely determined by times $t<t_{0}$. In our version of the definition, this special property is included in the choice of test functions. Roughly speaking, the admissibily of a test function is determined by its behavior before the time $t_{0}$ and there is no condition imposed on what happens after that. It is not surprising that this refined definition turns out to be essentially equivalent to the usual one. However, having another definition makes the use of viscosity solutions more flexible. We will demonstrate this by an example in the last section of this note.

This paper is organized as follows. In section 2, we give our definition and show that under reasonable assumptions it agrees with the usual one. Section 3 contains an application concerning the asymptotic behavior of the solutions of the so-called $p$-parabolic equation. In the proof, the new version of the definition is utilized in an essential way.

Received by the editors August 23, 1999 and, in revised form, February 2, 2000.

2000 Mathematics Subject Classification. Primary 35K55, 35D99; Secondary 35B40.

Key words and phrases. Viscosity solutions, parabolic equations.

(C)2001 American Mathematical Society 


\section{THE REFINED DEFINITION}

Let $F \in C\left(\mathcal{D} \times \mathbb{R} \times \mathbb{R}^{n} \times S_{n}\right)$, where $\mathcal{D} \subset \mathbb{R}^{n+1}$ is open and $S_{n}$ denotes the space of $n \times n$ symmetric matrices. $F$ is called proper if

$$
F(x, t, s, p, X) \leq F(x, t, r, p, Y) \quad \text { for } Y \leq X, s \leq r .
$$

This is a standard assumption in the theory of viscosity solutions that, among other things, guarantees the consistency of the notion. Namely, it implies that a smooth function is a viscosity solution if and only if it satisfies the equation in the classical sense.

Our definition of a viscosity solution looks very much like the usual one. The only difference is that we significantly increase the class of test functions by ignoring what happens after the given time $t_{0}$. The standard definition is omitted here and we instead refer to [C] or [CIL, §8]. For clarity, we call a function that solves the equation in the sense of the refined definition below a parabolic viscosity solution.

Definition. An upper semicontinuous function $u: \mathcal{D} \rightarrow \mathbb{R}$ is a parabolic viscosity subsolution of the equation $u_{t}+F\left(x, t, u, D u, D^{2} u\right)=0$ in $\mathcal{D} \subset \mathbb{R}^{n+1}$ if

$$
\varphi_{t}\left(x_{0}, t_{0}\right)+F\left(x_{0}, t_{0}, \varphi\left(x_{0}, t_{0}\right), D \varphi\left(x_{0}, t_{0}\right), D^{2} \varphi\left(x_{0}, t_{0}\right)\right) \leq 0,
$$

whenever $\left(x_{0}, t_{0}\right) \in \mathcal{D}$ and $\varphi \in C^{2}(\mathcal{D})$ are such that $u\left(x_{0}, t_{0}\right)=\varphi\left(x_{0}, t_{0}\right)$ and $u(x, t) \leq \varphi(x, t)$ for all $(x, t) \in \mathcal{D}, t<t_{0}$. Similarly, a lower semicontinuous function $u: \mathcal{D} \rightarrow \mathbb{R}$ is a parabolic viscosity supersolution of $u_{t}+F\left(x, t, u, D u, D^{2} u\right)=0$ in $\mathcal{D}$ if

$$
\varphi_{t}\left(x_{0}, t_{0}\right)+F\left(x_{0}, t_{0}, \varphi\left(x_{0}, t_{0}\right), D \varphi\left(x_{0}, t_{0}\right), D^{2} \varphi\left(x_{0}, t_{0}\right)\right) \geq 0,
$$

whenever $\left(x_{0}, t_{0}\right) \in \mathcal{D}$ and $\varphi \in C^{2}(\mathcal{D})$ are such that $u\left(x_{0}, t_{0}\right)=\varphi\left(x_{0}, t_{0}\right)$ and $u(x, t) \geq \varphi(x, t)$ for all $(x, t) \in \mathcal{D}, t<t_{0}$. Finally, a continuous function $u: \mathcal{D} \rightarrow \mathbb{R}$ is a parabolic viscosity solution of $u_{t}+F\left(x, t, u, D u, D^{2} u\right)=0$ if it is both a parabolic viscosity subsolution and a parabolic viscosity supersolution.

In Theorem 1 below, we show that in most cases this refined definition is equivalent to the usual one. The one additional requirement we have on the equation is the following technical condition which is needed to ensure that the comparison principle holds. We assume that

there exists a function $\omega:[0, \infty) \rightarrow[0, \infty), \omega(0+)=0$, such that

$$
F\left(y, t, r, \frac{x-y}{\varepsilon}, Y\right)-F\left(x, t, r, \frac{x-y}{\varepsilon}, X\right) \leq \omega\left(|x-y|+\frac{1}{\varepsilon}|x-y|^{2}\right)
$$

$$
\text { whenever }(x, t),(y, t) \in \mathcal{D}, r \in \mathbb{R} \text { and } X, Y \in S_{n} \text { are such that }
$$

$$
-\frac{3}{\varepsilon}\left(\begin{array}{cc}
I & 0 \\
0 & I
\end{array}\right) \leq\left(\begin{array}{cc}
X & 0 \\
0 & -Y
\end{array}\right) \leq \frac{3}{\varepsilon}\left(\begin{array}{cc}
I & -I \\
-I & I
\end{array}\right) .
$$

It can be quite easily seen that equations of the type $u_{t}+F\left(u, D u, D^{2} u\right)=0$ where $F$ is proper always satisfy the above condition (see CIL $\S 3]$ ).

Theorem 1. Let $u \in U S C(\mathcal{D})$ and assume that $F$ is proper and $(*)$ holds. Then $u$ is a parabolic viscosity subsolution of $(\mathrm{P})$ if and only if it is a viscosity subsolution in the usual sense.

Theorem 1 is essentially a consequence of the following comparison principle (see C], CIL]). 
Theorem. Let $\Omega \subset \mathbb{R}^{n}$ be open and bounded, and assume that

$$
F \in C\left(\bar{\Omega} \times[0, T] \times \mathbb{R} \times \mathbb{R}^{n} \times S_{n}\right)
$$

is proper and $(*)$ holds. If $u$ and $v$ are a viscosity subsolution and a supersolution, respectively, of $(\mathrm{P})$ in $\Omega \times(0, T)$ such that $u \leq v$ on the parabolic boundary, then $u \leq v$ in $\Omega \times[0, T)$.

Proof of Theorem 1. It is clear that a parabolic subsolution is a subsolution in the usual sense. For the converse, we argue by contradiction and assume that there exist $\left(x_{0}, t_{0}\right) \in \mathcal{D}$ and $\varphi \in C^{2}(\mathcal{D})$ such that $0=(u-\varphi)\left(x_{0}, t_{0}\right) \geq(u-\varphi)(x, t)$ for all $(x, t) \in \mathcal{D}, t<t_{0}$, and

$$
\varphi_{t}\left(x_{0}, t_{0}\right)+F\left(x_{0}, t_{0}, \varphi\left(x_{0}, t_{0}\right), D \varphi\left(x_{0}, t_{0}\right), D^{2} \varphi\left(x_{0}, t_{0}\right)\right) \geq \varepsilon>0
$$

for some $\varepsilon$. Let $S_{r}=B_{r}\left(x_{0}\right) \times\left(t_{0}-r, t_{0}\right), r>0$, and let $\partial_{p} S_{r}$ denote its parabolic boundary. Define $\tilde{\varphi}_{r}=\varphi+\left|x-x_{0}\right|^{4}+\left(t_{0}-t\right)^{4}-r^{4}$ and observe that since

$$
h(r)=\inf _{(x, t) \in S_{r}}\left\{\left(\tilde{\varphi}_{r}\right)_{t}+F\left(x, t, \tilde{\varphi}_{r}, D \tilde{\varphi}_{r}, D^{2} \tilde{\varphi}_{r}\right)\right\}
$$

is continuous with respect to $r, h(r) \geq \varepsilon / 2$ for $r>0$ small enough. In other words, we can find $r$ so that $\tilde{\varphi}_{r}$ is a classical supersolution in $S_{r}$. Next note that $\tilde{\varphi}_{r} \geq u$ on $\partial_{p} S_{r}$ by the definitions. Since $u$ is a viscosity subsolution, the comparison principle implies that $\tilde{\varphi}_{r} \geq u$ in $S_{r}$, and in particular, that $\tilde{\varphi}_{r}\left(x_{0}, t_{0}\right) \geq u\left(x_{0}, t_{0}\right)$. This, however, contradicts the assumption $\varphi\left(x_{0}, t_{0}\right)=u\left(x_{0}, t_{0}\right)$, and hence the theorem is proved.

\section{Application}

In this last section, we give a short proof for the fact that the asymptotic limit, as $t \rightarrow \infty$, of a viscosity solution of the p-parabolic equation

$$
u_{t}-\operatorname{div}\left(|D u|^{p-2} D u\right)=0
$$

with time-independent boundary values is a viscosity solution to the corresponding elliptic equation. For simplicity, we restrict ourselves to the degenerate case $2<$ $p<\infty$. For the singular case, the definition of viscosity solutions has to be slightly modified (cf. [IS], [JLM]). Our proof of Theorem 2 below follows the ideas of [W] (see also [F, Chap. 6] and [SZ] for results of this type).

Assume that $\Omega \subset \mathbb{R}^{n}$ is a bounded domain whose boundary satisfies the property of positive geometric density, that is, there exists $\alpha \in(0,1)$ and $r_{0}>0$ such that for every ball $B_{r}(x)$ with $x \in \partial \Omega$ and $0<r<r_{0}$ we have $\left|\Omega \cap B_{r}(x)\right| \leq(1-\alpha)\left|B_{r}(x)\right|$. Let $u \in C(\bar{\Omega} \times[0, \infty))$ be a viscosity solution of

$$
\left\{\begin{aligned}
u_{t}-\operatorname{div}\left(|D u|^{p-2} D u\right) & =0 & & \text { in } \Omega \times(0, \infty), \\
u(x, t) & =g(x) & & \text { on } \partial \Omega \times(0, \infty), \\
u(x, 0) & =g(x) & & \text { on } \Omega \times\{0\},
\end{aligned}\right.
$$

with $g: \bar{\Omega} \rightarrow \mathbb{R}$ being continuous. The results in DiB Chap. III] imply that the family $\{u(\cdot, t): t \in(0, \infty)\}$ is equicontinuous. Since it is also uniformly bounded due to the boundedness of $g$, we infer from Ascoli-Arzelà's theorem that there exists a sequence $t_{j} \rightarrow \infty$ such that $u\left(\cdot, t_{j}\right)$ converge uniformly in $\bar{\Omega}$ to a function $U \in C(\bar{\Omega})$ for which $U(x)=g(x)$ for all $x \in \partial \Omega$.

We will need the following lemma, the proof of which only uses the homogeneity of the equation (see [DiB, p. 170]). 
Lemma. Let $u$ be as above. Then for every $(x, t) \in \Omega \times(0, \infty)$ and for $0<h<t$

$$
|u(x, t-h)-u(x, t)| \leq \frac{2\|g\|_{\infty, \Omega}}{p-2}\left(1-\frac{h}{t}\right)^{\frac{p-1}{2-p}} \frac{h}{t} .
$$

Theorem 2. Let $u$ be a viscosity solution of $(*)$ and assume that $\partial \Omega$ satisfies the property of positive geometric density. Then $u(x, t) \rightarrow U(x)$ as $t \rightarrow \infty$ uniformly in $\Omega$, and $U$ is the unique viscosity solution of the p-Laplace equation $-\operatorname{div}\left(|D U|^{p-2} D U\right)=0$ with the Dirichlet boundary condition $\lim _{y \rightarrow x} U(y)=g(x)$ for all $x \in \partial \Omega$.

Proof. Since it is known that the Dirichlet problem for the elliptic $p$-Laplace equation has a unique viscosity solution (see [JLM]), it is enough to show that $U$, the uniform limit of the sequence $u\left(\cdot, t_{j}\right)$, is a viscosity subsolution of the $p$-Laplace equation. For that, we fix $x_{0} \in \Omega$ and $\varphi \in C^{2}(\Omega)$ such that $0=U\left(x_{0}\right)-\varphi\left(x_{0}\right)>$ $U(x)-\varphi(x)$ for all $x \neq x_{0}$. Using the uniform convergence, we can find a sequence $x_{j} \rightarrow x_{0}$ such that $u\left(\cdot, t_{j}\right)-\varphi$ has a local maximum at $x_{j}$. Now let

$$
\varphi_{j}(x, t)=\varphi(x)+C\left(\frac{t}{t_{j}}\right)^{\frac{p-1}{2-p}} \frac{t_{j}-t}{t_{j}},
$$

where $C=2\|g\|_{\infty, \Omega} /(p-2)$. Then, using the lemma,

$$
\begin{aligned}
u\left(x_{j}, t_{j}\right)-\varphi_{j}\left(x_{j}, t_{j}\right) & =u\left(x_{j}, t_{j}\right)-\varphi\left(x_{j}\right) \geq u\left(x, t_{j}\right)-\varphi(x) \\
& \geq u(x, t)-\varphi(x)-C\left(1-\frac{t_{j}-t}{t_{j}}\right)^{\frac{p-1}{2-p}} \frac{t_{j}-t}{t_{j}} \\
& =u(x, t)-\varphi_{j}(x, t)
\end{aligned}
$$

for any $x \in \Omega$ and $t<t_{j}$. Hence $\varphi_{j}$ is, according to the refined definition, an admissible test function for $u$ at the point $\left(x_{j}, t_{j}\right)$. This implies that

$$
-\operatorname{div}\left(\left|D \varphi\left(x_{j}\right)\right|^{p-2} D \varphi\left(x_{j}\right)\right) \leq \frac{C}{t_{j}}
$$

and we conclude by letting $j \rightarrow \infty$.

Remarks. $(i)$ The functions $\varphi_{j}$ constructed in the proof above are in general nonadmissible as test functions according to the usual definition of viscosity solutions.

(ii) Theorem 2 could have been formulated using distributional weak solutions because in our case they coincide with the viscosity solutions. We refer the reader to JILM, where both the elliptic and the parabolic case are treated.

(iii) In $[\mathrm{KL}]$ it is shown that in the case of a space-time cylinder the regularity of a lateral boundary point for the $p$-parabolic equation can be characterized by a Wiener-type test. Using this, the assumption on the domain $\Omega$ in Theorem 2 can be relaxed considerably. In particular, if $p>n$, then the result holds for any domain.

\section{ACKNOWLEGEMENT}

This question was first proposed to the author by Juan J. Manfredi, University of Pittsburgh. The author was supported by the Academy of Finland, project 41964 . 


\section{REFERENCES}

[C] Crandall, M. G., Viscosity solutions: a primer, Viscosity solutions and applications (Montecatini Terme, 1995), Lecture Notes in Math., 1660, Springer, Berlin (1997), 1-43. MR 98g:35034

[CIL] Crandall, M. G., H. Ishii, and P-.L. Lions, User's guide to viscosity solutions of second order partial differential equations, Bull. Amer. Math. Soc. 27 (1992), 1-67. MR 92j:35050

[DiB] DiBenedetto, E., Degenerate parabolic equations, Springer-Verlag, New York (1993). MR 94h:35130

[F] Friedman, A., Partial differential equations of parabolic type, Prentice-Hall, Inc., Englewood Cliffs, N.J. (1964). MR 31:6062

[IS] Ishii, H., and P. E. Souganidis, Generalized motion of noncompact hypersurfaces with velocity having arbitrary growth on the curvature tensor, Tôhoku Math. J. 47 (1995), 227-250. MR 96e:35069

[JLM] Juutinen, P., P. Lindqvist, and J. Manfredi, On the equivalence of viscosity solutions and weak solutions for a quasilinear equation, manuscript (2000).

[KL] Kilpeläinen, T., and P. Lindqvist, On the Dirichlet boundary value problem for a degenerate parabolic equation, SIAM J. Math. Anal. 27 (1996), 661-683. MR 97b:35118

[SZ] Sternberg, P., and W. P. Ziemer, Generalized motion by curvature with a Dirichlet condition, J. Differential Equations 114 (1994), 580-600. MR 96a:35096

[W] Wu, Y., Absolute minimizers in Finsler metrics, Ph.D. dissertation, UC Berkeley (1995).

Department of Mathematics, University of Jyväskylä, P.O. Box 35 (MaD), FiN-40351, JYVÄSKYLÄ, FinLAND

E-mail address: peanju@math.jyu.fi 